\section{Dendrimer throws a blanket on RA}

\author{
By Kai-Jye Lou, Staff Writer
}

A research group in France has developed an azabisphosphonatecapped dendrimer that blocks inflammation, cartilage degradation and bone erosion in mouse models of rheumatoid arthritis. ${ }^{1}$ The group is getting the compound ready for clinical trials.

Dendrimers are highly branched, large molecules that have been evaluated as imaging agents, drug delivery vehicles and therapeutics. A dendrimer's properties are primarily controlled by functional groups on its surface, but other parts of the molecule also can contribute.

The French team, led by researchers at a Institut National de la Santé et de la Recherche Médicale (INSERM) lab affiliated with Paul Sabatier University and the Centre National de la Recherche Scientifique (CNRS), had previously synthesized a dendrimer containing a cyclotriphosphazene core and phenoxymethyl-methylhydrazone branches capped with azabisphosphonate functional groups. The team showed that its dendrimer was internalized by monocytes and could have anti-inflammatory properties, ${ }^{2,3}$ but neither the mechanism nor the therapeutic effects were explored.

Now, the group has elucidated the dendrimer's mechanism of action

Figure 1. Targeting rheumatoid arthritis on multiple fronts. Rheumatoid arthritis is an autoimmune disease characterized by inflammation, cartilage degradation and bone erosion. Hayder et al. report that their azabisphosphonate-capped dendrimer could attenuate RA pathology on all three fronts.

On the inflammation front, when monocytes internalize the dendrimer, they show decreased expression of colony-stimulating factor 1 receptor (CSF1R; C-FMS; CD115). This leads to increased secretion of anti-inflammatory T helper type 2 (Th2) cell cytokines such as IL-4 and IL-10 and lower secretion of proinflammatory Th1 cell cytokines like IL-1 $\beta$, tumor necrosis factor- $\alpha$ (TNF- $\alpha$ ), IL-2, IL-6, IL-17 (IL-17A) and interferon- $\gamma$ (IFNG; IFN- $\gamma$ ) [a].

On the bone remodeling front, dendrimer-mediated inhibition of C-FMS expression prevents macrophage colony-stimulating factor 1 (CSF1; M-CSF) from promoting the differentiation of monocytes into precursors of bone-resorbing osteoclasts, which express tumor necrosis factor receptor superfamily member 11a (TNFRSF11A; RANK; CD265) [b(1)]. In these precursors, RANK activation by receptor activator of NF-KB ligand (RANKL; TNFSF11) promotes differentiation into osteoclasts. Moreover, the dendrimer also prevents myeloid dendritic cells from undergoing transdifferentiation into osteoclasts [b(2)].

Finally, the dendrimer decreases serum levels of matrix metalloproteinase 3 (MMP3) and MMP9, which are responsible for cartilage degradation $[\mathbf{b}(3)]$. Although this mechanism remains unclear, the researchers speculate that dendrimer-mediated inhibition of $\mathrm{C}$-FMS expression reduces the expression of these enzymes by inhibiting monocyte proliferation. via studies in patient tissue samples and mouse models (see Figure 1, "Targeting rheumatoid arthritis on multiple fronts").

In two mouse models of RA, the dendrimer decreased levels of inflammatory cytokines while increasing levels of anti-inflammatory cytokines, resulting in lower inflammation than no treatment.

The molecule also inhibited the generation of osteoclasts, which carry out bone resorption, and decreased the levels of enzymes that carry out cartilage degradation, leading to less RA-associated bone erosion and cartilage destruction than those in controls.

The effects were unique to the dendrimer-two other dendrimers with either different functional groups or a different core and branches showed no therapeutic effects in the models.

The dendrimer similarly lowered markers of inflammation and bone erosion in synovial tissue samples from RA patients.

The key target underlying the dendrimer's effects was found to be colony-stimulating factor 1 receptor (CSF1R; C-FMS; CD115). C-FMS is the receptor for macrophage colony-stimulating factor 1 (CSF1; $\mathrm{M}-\mathrm{CSF}$ ) - a growth factor important for the generation of proinflammatory cells and osteoclasts.

Results were published in Science Translational Medicine.

"We show in our study that the therapeutic effect of our dendrimer is mediated by the inhibition of C-FMS on monocytes," said co-corresponding author Rémy Poupot, a professor in the Department of Biochemistry at Paul Sabatier University.

"This is a very important and thorough study, which has not only shown the clinical relevance of dendrimers in rheumatoid arthritis but also elucidated the effects of subtle dendrimer structural changes on efficacy," noted Rangaramanujam Kannan, a professor in the Department of Chemical Engineering and Materials Science at Wayne State University. "The dendrimers appear to be engineered toward both
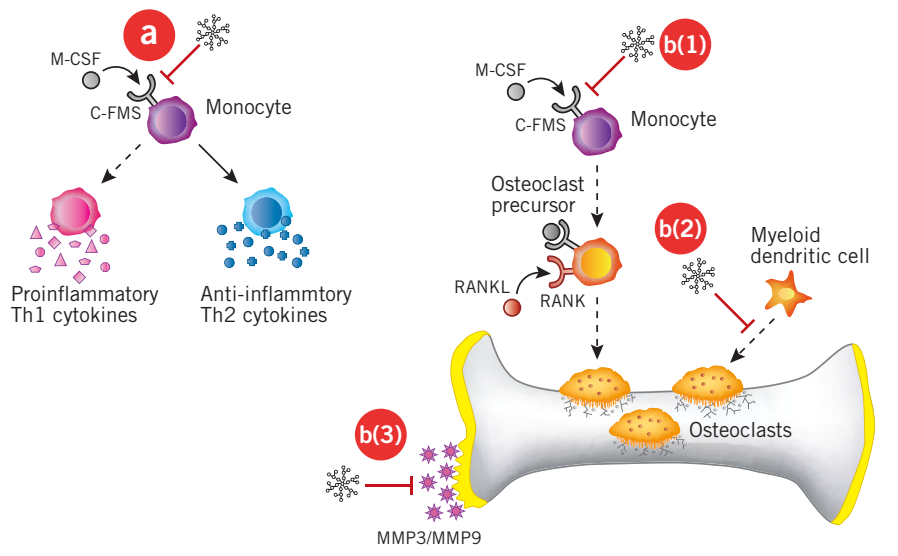


\section{ANALYSIS}

\section{TARGETS \& MECHANISMS}

anti-inflammatory effects and antiosteoclastogenic activity, bringing a bimodal attack toward rheumatoid arthritis."

Kannan added that dendrimers in general also have the advantage of being able to carry a large therapeutic payload, which could lead to lower drug doses and decreased side effects compared with using the drug itself. "Even though dendrimers appear to be facing higher structural purity expectations compared to other polymers, this study and others are starting to show the importance of the dendritic structure for medical applications," he told SciBX.

\section{Above the crowd}

Only one company, Plexxikon Inc. (now part of Daiichi Sankyo Co. Ltd.), has disclosed a clinical stage compound for RA that targets the interaction between C-FMS and M-CSF.

Plexxikon's PLX5622, an oral, small molecule, selective C-FMS inhibitor, is in Phase I testing, with data expected this quarter. In preclinical arthritis models, PLX5622 lowered inflammation, cartilage damage and bone resorption.

Pfizer Inc. was developing PD-360324, a mAb against M-CSF, but in its January 2010 pipeline report the company said it had discontinued the Phase I compound.

Although there are many RA drugs, Poupot and co-corresponding author Jean-Luc Davignon think the azabisphosphonate-capped dendrimer will have an efficacy edge because it directly blocks the generation of disease-associated cells that mediate inflammation and bone degradation.

Davignon is a director of research at CNRS.

"This is a big contrast to the anti-TNF drugs for arthritis," Davignon told SciBX. "While these drugs can reduce both inflammation and bone erosion, they target these disease processes in a less direct manner than our dendrimer."

He added that tumor necrosis factor (TNF) is just one of the proinflammatory cytokines known to drive RA pathology. In contrast, dendrimer-mediated inhibition of C-FMS expression on monocytes led to decreases in at least six proinflammatory cytokines, including TNF.
Nevertheless, Poupot thinks initial clinical trials of the dendrimer will likely be in patients who have failed anti-TNF therapies. He declined to provide a timeline for entering the clinic with the dendrimer but noted that an industry partner will be needed to take the compound into clinical trials.

The French group is now carrying out preclinical studies to assess the toxicity and tolerability of the dendrimer and determine whether the molecule can be delivered orally. Poupot expects to have toxicity data by October.

He added the group plans to start pharmacokinetic studies in the coming months and still needs to show that the dendrimer can be manufactured to current GMP standards and synthesized on an industrial scale.

INSERM, Paul Sabatier University and CNRS have cofiled two patent applications covering the biological properties and the synthesis of the azabisphosphonate-capped dendrimer. The compound is available for licensing.

Lou, K.-J. SciBX 4(20); doi:10.1038/scibx.2011.561

Published online May 19, 2011

\section{REFERENCES}

1. Hayder, M. et al. Sci. Transl. Med.; published online May 4, 2011; doi:10.1126/scitransImed.3002212

Contact: Jean-Luc Davignon, Institut National de la Santé et de la Recherche Médicale (INSERM), Toulouse, France

e-mail: jean-luc.davignon@inserm.fr

Contact: Rémy Poupot, same affiliation as above e-mail: remy.poupot@inserm.fr

2. Poupot, M. et al. FASEB J. 20, 2339-2351 (2006)

3. Fruchon, S. et al. J. Leukoc. Biol. 85, 553-562 (2009)

\section{COMPANIES AND INSTITUTIONS MENTIONED}

Centre National de la Recherche Scientifique, Toulouse, France Daiichi Sankyo Co. Ltd. (Tokyo:4568; Osaka:4568), Tokyo, Japan Institut National de la Santé et de la Recherche Médicale, Toulouse, France

Paul Sabatier University, Toulouse, France

Pfizer Inc. (NYSE:PFE), New York, N.Y.

Plexxikon Inc., Berkeley, Calif.

Wayne State University, Detroit, Mich. 